\title{
EDITORIAL
}

\section{REFLEXIONES EN TORNO AL MARCO EPISTEMOLÓGICO DE LA DISFORIA DE GÉNERO}

\author{
María Fernández Rodríguez (1,2), Patricia Guerra Mora (3), Eloya Martín Sánchez (4) y Grupo \\ GIDSEEN (*).
}

(1) Unidad de Tratamiento de Identidad de Género del Principado de Asturias (UTIGPA). Hospital San Agustín de Avilés

(2) Centro de Salud Mental I (CSMI) “La Magdalena”. Área sanitaria III.

(3) Hospital San Agustín de Avilés. Área sanitaria III.

(4) Eloya MS Psicología.

Grupo GIDSEEN: Mari Cruz Almaraz Almaraz (Andalucía), José Antonio Álvarez Diz (Asturias), Nuria Asenjo Araque (Madrid), Laura Audí Parera (Cataluña), Antonio Becerra Fernández (Madrid), Virginia Bellido Castañeda (País Vasco), Isabel Esteva (Andalucía), maría Fernández-Rodríguez (Asturias), María Gómez-Balaguer (Comunidad Valenciana), Esther Gómez-Gil (Cataluña), Felipe Hurtado (Comunidad Valenciana), Juan Pedro López-Siguero (Andalucía), María Jesús Lucio (Comunidad de Madrid), Juana Martínez-Tudela (Andalucía), Óscar Moreno-Pérez (Comunidad Valenciana), Jesús Pérez-Luis (Islas Canarias), Susana Ponce de León (País Vasco), Itxaso Rica (País Vasco), José Miguel Rodríguez Molina (Comunidad de Madrid), Carmen Sanisidro (Aragón), Marta Toni García (Navarra), Ángela Vidal Hagemeijer (Cataluña), Angelines Vidales Miguélez (Castilla y León), Aurelia Villar Bonet (Castilla y León).

El comienzo de la actividad asistencial en la priemra unidad española de identidad de género, en 1999 en Andalucía, se caracterizó por un modo de intervención basado en el modelo médico asistencial: evaluación, diagnóstico y tratamiento. La evaluación tenía como objetivo establecer un diagnóstico de transexualismo para poder iniciar un proceso transexualizador, es decir, la inclusión o no en la triada terapéutica (psicológica, hormonal y quirúrgica) $)^{(1,2)}$.

Por tanto, en primer lugar todos los equipos nacionales e internacionales asumían que la transexualidad ${ }^{(3)}$ o trastorno de la identidad de género $(\mathrm{TIG})^{(4)}$ entraba como una categoría diagnóstica de las Clasificaciones Internacio- nales de los trastornos mentales. El diagnóstico se fundamentaba en alteraciones en el modelo dicotómico tradicional (binarismo sexo/ género, hombre-masculino/mujer-femenina). Además, para establecer el diagnóstico de transexualismo se debía realizar el diagnóstico diferencial con otros TIG, con algunas parafilias, como el travestismo fetichista ${ }^{(5)}$ y descartar que el problema de identidad fuera un síntoma de un trastorno psicopatológico o de personalidad. Desde esta conceptualización podría considerarse el transexualismo como una psicopatología que precisaba una intervención médico asistencial, consideración que ha sido ampliamente criticada por los colectivos afectados.

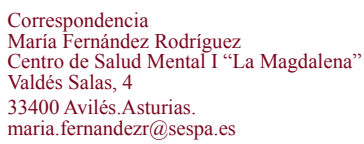

Cita sugerida: Fernández Rodríguez M, Guerra Mora P, Martín Sánchez E,
Grupo GIDSEEN. Reflexiones en torno al marco epistemológico de la dis-
foria de género. Rev Esp Salud Pública.2016; vol 90: 16 de agosto: e1-e4. 
Trabajar con este modelo asistencial resultaba cómodo y seguro para los profesionales. Tanto las Clasificaciones Diagnósticas como los Estándares Asistenciales (EA) de la Harry Benjamin International Gender Dysphoria Association (HBIGDA) proporcionaban referentes externos más o menos claros para incluir o excluir del proceso terapéutico. Además, desde un modelo dicotómico de la sociedad, los EA, hasta la sexta versión $^{(2)}$, establecían un modo de intervención procesual o por fases (triada terapéutica) que favorecía la consecución de objetivos terapéuticos. Este tipo de intervención estaba construido en base a un modelo dicotómico de la sociedad, que solo contemplaba dos sexos/géneros y donde "el verdadero transexual" perpetuaba este dimorfismo sexual y de género a través de sus demandas de reasignación sexual. Desde esta forma de proceder, además de mantener la tendencia de la sociedad a una "normalización" preestablecida (hombres masculinos y mujeres femeninas) se colocaba teóricamente a las personas afectadas en una situación de cierta subordinación y pasividad respecto a todo el proceso asistencial. El problema no se planteaba con aquellas que ya desde la infancia sentían y pensaban que pertenecían al sexo contrario a su sexo biológico ("transexuales primarios o genuinos"), que son la mayoría de las que consultan en las unidades de género,) y cuyo sentimiento $\mathrm{y}$ pensamiento se había hecho permanente a lo largo de los años, sino más bien con quienes desarrollaban una DG de comienzo más tardío ("transexuales secundarios") que además podía coexistir con otros trastornos psicopatológicos. La no inclusión de algunos de estos casos en el proceso terapéutico era vivida con gran malestar, conllevando la decisión clínica de una gran subjetividad, argumentando que nadie mejor que ellas (las personas usuarias) sabían lo que sentían y cuáles eran sus necesidades. Desde esta perspectiva el papel de la psicología clínica era de máxima responsabilidad, todo el proceso de cambio se derivaba de un diagnóstico preciso de transexualismo. Los usuarios cuestionaban el papel que tenía asignado el psicólogo clínico, que realizaba un deslizamiento del contexto terapéutico al contexto judicial. Durante los últimos años se produjo una mayor visibilidad de los temas relacionados con las variantes del género, llegando incluso a producirse una socialización de las distintas expresiones o manifestaciones del género. Por un lado, están todos los movimientos que defienden la despatologización ${ }^{(6-8)} y$, por otro, las críticas a una concepción de la sociedad exclusivamente binaria, donde empieza a reivindicarse $\mathrm{y}$, por tanto, a legislarse la libre autodeterminación del género $^{(9-10)}$. Los EA en su séptima versión ${ }^{(11)}$ y el DSM-5 ${ }^{(12)}$ ya habían recogido estas reivindicaciones, creando un nuevo marco teórico y de actuación. Los EA de esta última versión desarrollan un modelo asistencial que supera el dimorfismo sexual y de género, establece que no sólo hay dos géneros y que el género sentido y expresado no tiene por qué ir indisolublemente ligado al sexo biológico. Supera el modelo de asistencia basado en la triada terapéutica para atender las necesidades y las demandas de las personas que presentan diversidad de expresiones de género ${ }^{(13)}$. La nueva terminología de Disforia de Género ${ }^{(11,12)}$ deja de tener connotaciones psicopatológicas, la identidad en sí no es objeto de diagnóstico, solo el malestar que produce. La DG no solo está presente en las personas transexuales. Para las personas transexuales, transgénero y disconformes con su género, la identidad o expresión de género puede diferir del sexo natal. Un sujeto cumpliría el criterio de DG con un mínimo de características ( 2 de 6 ), como puede ser, un fuerte deseo de ser del otro sexo o de ser tratado como del otro sexo (o de un sexo alternativo distinto al que se le asigna). Interesante es este planteamiento del DSM-5, que, al igual que los EA, rompe con el binarismo sexo/género hasta ahora hegemónico e introduce la posibilidad de identificarse con un sexo/género alternativo y que no se limita a la categoría de hombre y mujer o femenino y masculino. En estos momentos, con estas premisas el modelo asistencial de partida se 
derrumba y algunos facultativos quedan atrapados en un pensamiento dicotómico de la sociedad, en un binarismo sexo/género que abarca tanto la concepción de la identidad como de la orientación. Otros equipos intentan consensuar las decisiones terapéuticas con los supuestos que no se beneficiarían de intervenciones farmacológicas o quirúrgicas y que serían subsidiarios de otras terapias (cosméticas, documentales, etc) ${ }^{(14)}$.

En los últimos años a las unidades de atención a la DG están llegando personas cuya identidad, expresión de género y orientación no se fundamenta en los dos géneros/sexos hegemónicos, cuestionan la dicotomía hombre/ mujer. Surge también una nueva terminología para definir la orientación de las personas que no reconocen la existencia de un género asociado a un sexo, pansexualidad. Un sujeto pansexual no tiene en cuenta ni el sexo ni el género, simplemente siente atracción por las personas. Las clasificaciones diagnosticas predecesoras al DSM-5 establecían que se puede sentir atracción por los hombres, por las mujeres, por ambos o por ninguno. Curiosamente el DSM-5 con DG ya no hace alusión a la orientación sexual.

En este contexto aumenta la incertidumbre del clínico cuyo nuevo modelo teórico externo y la posibilidad de elección terapéutica aún no están definidas. La demanda ocupa un lugar prioritario a la vez que disminuye el margen de actuación del clínico. Recientemente la Asociación Americana de Psicologíaha elaborado un documento sobre las recomendaciones para la práctica psicológica con personas transgénero y disconformes con el género ${ }^{(15)}$. En él destaca la importancia de adoptar un enfoque interdisciplinar para la atención integral. El trabajo en equipo asegura una mayor calidad asistencial al proporcionar una continuidad de cuidados y no continuados. No debemos olvidarnos que los equipos de atención a la DG están situados e integrados en contextos sociosanitarios predeterminados. Desde este encasillamiento deben esforzarse en la comprensión y aceptación de las distintas identidades y de las expresiones o manifestaciones de género que superan el concepto binario de la sociedad. La actitud de los profesionales hacia la identidad y las distintas expresiones del género va a ser decisiva en la atención prestada tanto a los personas usuarias como a sus familias. De aquí surge la importancia y la necesidad de las unidades interdisciplinares especializadas en la atención a la DG. La formación continuada que se imparte a los profesionales de salud mental y de endocrinología con respecto a los temas relacionados con el género y sus manifestaciones es muy escasa. Estos contenidos tampoco suelen formar parte de los programas formativos universitarios ni de la formación sanitaria especializada. En los últimos años, las Unidades de Género han partido ya de los principios básicos de despatologización y del derecho a la autodeterminación del género y consideran que el apoyo psicológico puede aportar beneficios en todo el proceso identitario. El trabajo del psicólogo clínico dentro de estas unidades especializadas debe superar el planteamiento evaluador para focalizar su intervención en la prevención y promoción de la salud. Las personas trans hacen referencia a sus desventajas sociales en un mundo dicotómico donde las barreras sociales y actitudinales son evidentes. El acompañamiento psicológico puede favorecer el abordaje y la superación de los problemas de la vida diaria a través de la elaboración de nuevas estrategias de afrontamiento.

Se requiere una reflexión sobre el abordaje de las nuevas variantes de género y considerar su inclusión social sin adoptar necesariamente el modelo de tratamiento integral (hormonal o quirúrgico). Estamos en un nuevo marco teórico, más inclusivo y respetuoso con la diversidad de género que forma parte del contexto social y que nos está exigiendo salir de nuestros compartimentos estancos y modificar nuestra epistemología.

\section{BIBLIOGRAFÍA}

1. The Harry Benjamin International Gender Dysphoria Association-HBIGD. The standards of care for gender identity disorders. $5^{\mathrm{a}}$ version. Mineapolis: HBIGDA; 1998. 
3. Organización Mundial de la Salud-OMS (1992). Clasificación Internacional de Enfermedades $10^{\mathrm{a}}$ ed. CIE-10. Ginebra: WHO; 1992.

4. American Psychiatric Association-APA . Manual Diagnóstico y Estadístico de los trastornos mentales. $4^{\mathrm{a}}$ ed. rev. DSM-IV-TR. Washington, DC: APA. Barcelona: Masson; 2002.

5. Fernández Rodríguez M, Guerra Mora P y GarcíaVeg, E. ¿Es posible distinguir el transvestismo del transexualismo? C Med Psicosom. 2012;102: 24-38.

6. Suess A. La despatologización trans desde una perspectiva de derechos humanos. Ponencia presentada en Jornadas Feministas Estatales. Granada: Jornadas Feministas Estatales;diciembre 2009. Disponible en www.feministas.org/jornadas.html.

7. WPATH Board of Directors. De-psychopathologisation statement released May 26, 2010. (Citado en enero de 2012). Disponible en: de http://wpath.org/

8. STP. Campaña Internacional Stop Trans Pathologization. Reflexiones de STP sobre el proceso de revisión de la CIE y la publicación del DSM-5; Disponible en: http://www.stp2012.info/Comunicado STP agosto2013.pdf

9. Boletín Oficial del Estado. Ley $2 / 2014$, de 8 de julio, integral para la no discriminación por motivos de identidad de género y reconocimiento de los derechos de las personas transexuales de Andalucía. BOE núm 193 de 9 de agosto de 2014.

10. Boletín oficial de la Asamblea de Madrid. Ley de Identidad y Expresión de Género e Igualdad Social y no Discriminación de la Comunidad de Madrid. Boletín oficial de la Asamblea de Madrid, num 51. 21 de marzo de 2016.

11. The World Professional Association for Transgender Health-WPATH Standards of Care for the Health of Transsexual, Transgender, and Gender Nonconforming People, $7^{\mathrm{a}}$ version; 2011. Disponible en http:// www.wpath.org/publications_standards.cfm

12. American Psychiatric Association-AP. Diagnostic and statistical manual of mental disorders, $5^{\mathrm{a}}$ edDSM-5. Arlington: American psychiatric Publishing; 2013.

13. Fernández Rodríguez M, Guerra Mora P y GarcíaVega E. La $7^{\mathrm{a}}$ versión de los Estándares Asistenciales de la WPATH. Un enfoque diferente que supera el dimorfismo sexual y de género. Rev Asoc Esp Neurop. 2014; 234(122): 317-335.
14. Asiain Vierge S, Gorneman Schaffer I, Giraldo Ansio F, Lara Montenegro J, Esteva de Antonio I, Gómez Banovio M y Bergero Miguel T. Una reflexión sobre el concepto de género alrededor de la transexualidad. Rev Asoc Esp Neuropsiq. 2008; XXVIII (101): 211226.

15. American Psychological Association- APA. Guidelines for Psychological Practice with transgender and gender nonconforming people; 2015. Disponible en https:// www.apa.org/practice/guidelines/transgender.pdf 